\title{
Chemical Constituents and Anti-Ulcer Activity of Ethylacetate Extract of the Leaves of Sanchezia nobilis Hook.F.
}

\author{
Loi Vu Duc ${ }^{1, *}$, Xuan Bui Thi ${ }^{1}$, Ngoc Tran Minh ${ }^{2}$
}

\section{Loi Vu Duc ${ }^{1, *}$, Xuan Bui Thi', Ngoc Tran Minh ${ }^{2}$}

'School of Medicine and Pharmacy, Viet nam National University, Hanoi, VIETNAM. ${ }^{2}$ National Institute of Medicinal Materials, VIETNAM.

\section{Correspondence}

\section{Loi Vu Duc}

School of Medicine and Pharmacy, Vietnam National University, No.144 Xuan Thuy street, Cau Giay, Hanoi, VIETNAM.

E-mail: ducloi82@gmail.com

History

- Submission Date: 11-05-2019.

- Review completed: 28-05-2019;

- Accepted Date: 04-06-2019.

DOI : 10.5530/pj.2019.11.182

Article Available online

http://www.phcogj.com/v11/i6

\section{Copyright}

(C) 2019 Phcogi.Com. This is an openaccess article distributed under the term of the Creative Commons Attribution 4.0 International license.

\begin{abstract}
Study have two objectives. First objective is about the identification of the five components The second objective is about the antiulcer activity of the ethylacetate extract. Five compounds (1-5) were isolated from the leaves of Sanchezia nobilis Hook.F. collected in Nam Dinh province by chromatographic methods. These compounds were identified as: Quercetin (1), Scopoletin (2), Kaempferol-3-O- $\alpha$-L-rhamnopyranosyl-(1 $\rightarrow 6)-\beta$-D-glucopyranoside (3), Quercetin-3-O- $\alpha$-L-rhamnosyl-( $1 \rightarrow 6)-\beta$-D-glucopyranosyl-( $1 \rightarrow 3)-\beta$-D-glucopyranoside (4), 3'-Omethyl-3, 4-methylenedioxy ellagic acid (5). Their structures were elucidated by spectroscopic methods, including mass spectrometry (MS) and nuclear magnetic resonance (NMR). Compounds 3, 4, 5 were isolated from the leaves of Sanchezia nobilis Hook.F. for the first time. Evaluation the effect of gastric and duodenal anti-ulcer on cyteamine induced gastric ulcer models indicates at this ethylacetate extract has the effect to improve the extent of ulcer lesions treatment $(54,17 \%)$; obviously reduced the average of number pocket of ulcers $(1,85 \pm$ $0,80)$ and ulcer index $(5,61 \pm 2,69)$, however, it does not change the area of the ulcer.

Key words: Sanchezia nobilis, Quercetin, Scopoletin, Anti-ulcer activity
\end{abstract}

\section{INTRODUCTION}

Sanchezia nobilis Hook.F. is being paid attention and studied in a lot of different ways. The research of Abu Shuaib Rafshanjani and partners about the evaluation of the safety of Brine shrimp lethality bioassay method on $n$-hexane and ethylacetate segments, the results showed that both segments were safer than vincristine sulphate. ${ }^{1}$ With antimicrobial, antifungal and insecticidal testing by diffusion on agar plates with 15 strains of Gram $(+)$ and Gram (-) bacteria; 6 strains of fungus and insects Tribolium castaleum was not as good as comparison antibiotics. ${ }^{2}$ Mohammad javad Paydar and co-worker have tried the antioxidant effect by Oxygen Radical Antioxidant Capacity (ORAC) method, the results were nearly equal to Quercetin and anti-cancer on methanolic segment from Sanchezia speciosa extract on MCF-7, SKMEL-5, HUVEC giving the best results on CMF7 cell lines, the anti-cancer effect on Hela cells from the roots of Sanchezia nobilis produced good results by MTT of Nusrat Shaheen and co-worker., ${ }^{3,4}$ Research on the antioxidant effect of DPPH and anti-inflammatory by inhibition of albumine denaturation assay method by Bui Thanh Tung and partners gave positive results. ${ }^{5}$ Some new claims showing that Sanchezia nobilis Hook.F. contains a number of substance groups such as flavonoids, glycosides, carbohydrates, alkaloids, steroids, phenolic, saponins and tannins. Some specific substances were isolated such as 5 matsutake alcohol compounds, in which the four compounds were first isolated from the Acathanceae family and one was the first isolated from nature, 3-O-arabarabinopyranosyl-(1-6) - $\beta$-glucopyranosyl-(1$6)$ - $\beta$-glucopyranosyl-1-octen-3-ol and 6 other compounds from the leaf and root methnol extract, in which 1 substance was first isolated from naturally, 9-O- $\beta$-xylopyranosyl- $(1 \rightarrow 6)-O-\beta$ glucopyranosyl- $(1 \rightarrow 6)-O-\beta$-glucopyranosyltranscinnamyl alcohol, the 4 substances were first isolated from the Acanthaceae family and 3 substances were reported for the first time from Sanchezia by Ahmed and co-worker Bui Thanh Tung and partners also isolated 4 compounds in which 3 compounds were first isolated from Sanchezia speciosa. ${ }^{6-8}$ In Vietnam, Sanchezia are found in many localities such as Tuyen Quang, Quang Nam, Da Nang, Nam Dinh, Vinh Phuc, Phu Tho and Thai Nguyen. ${ }^{9}$ In Vietnam, many people used Sanchezia nobilis Hook.F. as a traditional precious medicine to cure gastritis. We need more research on Sanchezia nobilis Hook.F. to use them more effective.

\section{MATERIALS AND METHODS}

\section{Plant material}

The leaves of Sanchezia nobilis Hook.F. were collected in Co Le hamlet, Truc Ninh district, Nam Dinh province, Viet Nam, during January, 2018 and authenticated by the School of Medicine and Pharmacy, Vietnam National University, Hanoi, Vietnam (No: 190DV18 SMP-VNU). A voucher specimen has been deposited in the SMP-VNU.

\section{General experimental procedures}

Melting points were measured on Mikroskopheiztisch PHMK-50 (VEB WaegetechnikRapido, Germany). The FT-IR spectra were recorded on an IMPACT410FT-IR spectrometer (CARL ZEISS JENA). The NMR $\left[{ }^{1} \mathrm{H}(500 \mathrm{MHz}),{ }^{13} \mathrm{C}(125 \mathrm{MHz})\right.$, and DEPT90 and $135 \mathrm{MHz})$ ] spectra were recorded on an

Cite this article: Duc LV, Thi XB, Minh NT. Chemical constituents and anti-ulcer activity of ethylacetate extract of the leaves of Sanchezia nobilis Hook.F. Pharmacog J. 2019;11(6):117280. 
AVANCE spectrometer AV 500 (Brucker, Germany) in the Institute of Chemistry, Vietnam Academy of Science and Technology (VAST). Chemical shifts were reported in ppm downfield from TMS with J in Hz. Electrospray Ionization Mass Spectra (ESI-MS) were recorded on a Varian Agilent 1100 LCMSD mass spectrometer. Optical rotation was measured on WXG-4 disc polarimeter. Analytical TLC was performed on Kieselgel 60 F254 (Merck) plates (silica gel, $0.25 \mathrm{~mm}$ layer thickness) and RP-18 $\mathrm{F}_{254}$ (Merck) plates (0.25 mm layer thickness). Spots were visualized using ultraviolet radiation (at 254 and $365 \mathrm{~nm}$ ) and by spraying with $10 \% \mathrm{H}_{2} \mathrm{SO}_{4}$, followed by heating with a heat gun. Column chromatography was performed on silica gel (70-230 and 230-400 mesh, Merck). Organic solvents were of analytical grade. Optical densities were read on an ELISA plate reader (Bio-rad)

\section{Extraction and isolation}

The leaves of Sanchezia nobilis Hook.F. were washed, sun dried and cut into small pieces. The plant powder $(2.5 \mathrm{~kg})$ was extracted with ethanol $80 \%(8 \mathrm{~L})$ for three days at room temperature, got the first aqueous extract. Repeated the extraction twice by adding more solvent to make ingredient inside the solvent at least $2-3 \mathrm{~cm}(8 \mathrm{~L} /$ time $)$, got the second and third aqueous extract.

The extracts were filtered with filter paper, combined, and evaporated under low pressure resulting in a semi solid crude extract $(150 \mathrm{~g})$.

The ethanol crude extract (100 g) was suspended in water and successively partitioned with $\mathrm{n}$-hexane and ethylacetate $(3 \times 500 \mathrm{~mL}$, each solvent/30 minutes). Combined solvent $n$-hexane and ethylacetate were then evaporated under low pressure to obtain the $n$-hexane and the ethylacetate fractions denoted by $\mathrm{H}(9.2 \mathrm{~g})$ và $\mathrm{E}(28.8 \mathrm{~g})$, respectively. The remaining water extract was evaporated the solvent by heat to give water fraction denoted by $\mathrm{N}(26.6 \mathrm{~g})$

Conduct an ethylacetate bite analysis $(25.0 \mathrm{~g})$ on the chromatographic column of silica gel with a gradually increasing polarization solvent system consisting of $\mathrm{n}$-hexane:ethylacetate $(5: 1 \rightarrow 1: 1, \mathrm{v} / \mathrm{v}$, each $600 \mathrm{~mL}$ segment) and then chloroform:methanol $(10: 1 \rightarrow 1: 1, \mathrm{v} / \mathrm{v}$, each $500 \mathrm{~mL}$ segment) obtained 6 segments marked as E1 E6. Fraction E1 $(8.1 \mathrm{~g})$ was further separated on a silica gel column chromatography ( $\Phi 45 \mathrm{~mm}$ $\times 350 \mathrm{~mm}$ ), eluting with the mobile phase chlorofrom:methanol (40:1, $30: 1,20: 1,10: 1,5: 1)$ to get six fractions E1.1-E1.6. Fraction E3 $(4,2$ g) was chromatographed on a reversed-phase chromatography YMCRP-18, eluting with a solvent system of acetone:water (1:6, v/v). Inspect the elution tubes with thin-layer chromatography, combine the tubes and distillate the solvents to obtain small symbol sections: E3.1, E3.2, E3.3, E3.4. Fraction E3.2 was further separated on a silica gel column chromatography, eluting with chloroform:methanol $(15: 1, \mathrm{v} / \mathrm{v})$ to yield compound 1 (32 mg). Fraction E3.3 was further separated on a silica gel column chromatography, eluting with n-hexane:ethylacetate $(2: 1, \mathrm{v} / \mathrm{v})$ to yield compound 2 (24 mg). Fraction E4 (3.3 g) was further separated on a silica gel column chromatography, eluting with chlorofrom:methanol $(40: 1,30: 1,20: 1,10: 1,5: 1)$ to get five fractions E4.1-E4.5. Fraction E4.1 (0.8 g) was further separated on a silica gel column chromatography RP-18, eluting with water:methanol (10:1, $\mathrm{v} / \mathrm{v})$ to yield yellow powder $3(21 \mathrm{mg})$. Fraction E4.2 (0.6 g) was further separated on a silica gel column chromatography, eluting with ethylacetate:methanol $(3: 1, \mathrm{v} / \mathrm{v})$ to yield yellowish crystalline solid 4 (16 mg). Fraction E4.3 was further separated on a silica gel column chromatography, eluting with ethylacetate:methanol $(10: 1, \mathrm{v} / \mathrm{v})$ to yield compound 5 (14 mg) (Figure 1).

\section{Evaluation of anti-ulcer activity}

Gastric and duodenal anti-ulcer activity of ethylacetate fraction from Sanchezia nobilis Hook.F. was evaluated on cysteamine induced gastric and duodenal ulcer models in adult Wistar albino rats with two oral doses of cysteamine $(400 \mathrm{mg} / \mathrm{kg})$ at an interval of 4 h. ${ }^{10,11}$ Cysteamine hydrochloride has been found to be the most potent agent for inducing duodenal ulcer, and cysteamine induced duodenal ulcer in animals is now used to study the antiulcer activity of drugs. The cysteamine used in experimental studies has been found to concentrate in the duodenum. Its ulcerogenic effect may be due to the generation of reactive oxygen species (ROS), the decreasing defense activity of superoxide dismutase (SOD) and increasing duodenal endothelin-1 concentration, which are all associated with decreased duodenal mucosal blood flow. Oxidative stress enhanced free-radical levels, and an impaired in-cell antioxidant pool are important factors underlying the pathophysiologic mechanisms in a variety of diseases. Ranitidine reduces the amount of acid your stomach makes. Ranitidine is taken to prevent and treat stomach ulcers (Figure 2).

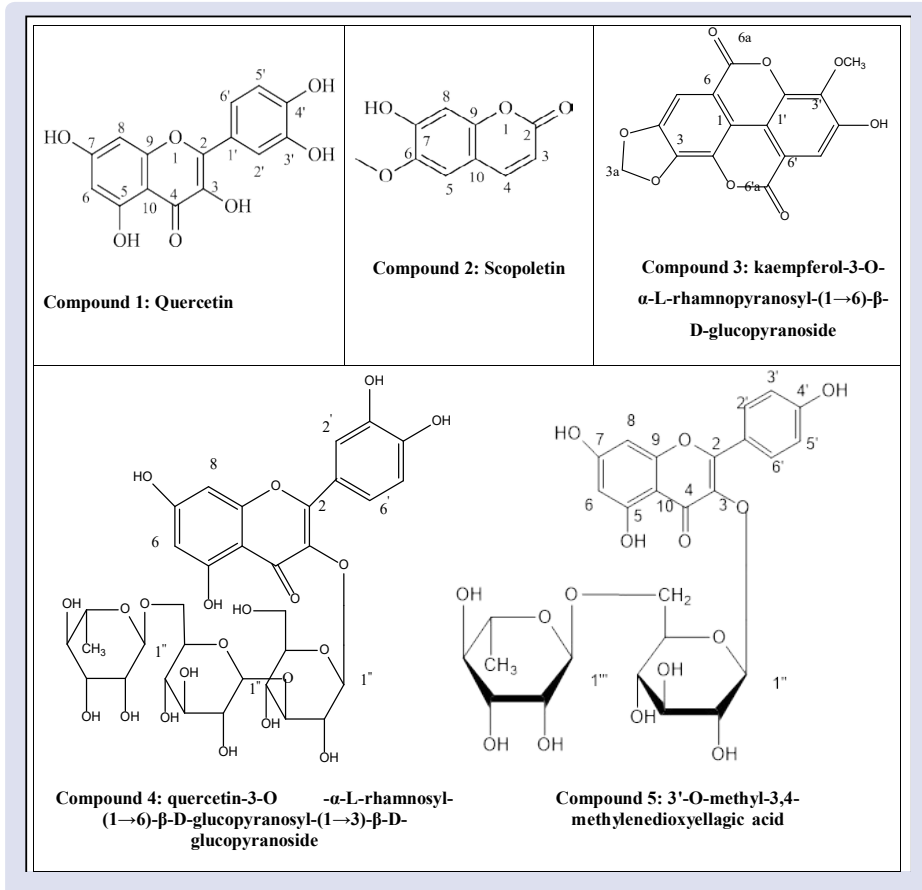

Figure 1: The compounds' structures. 


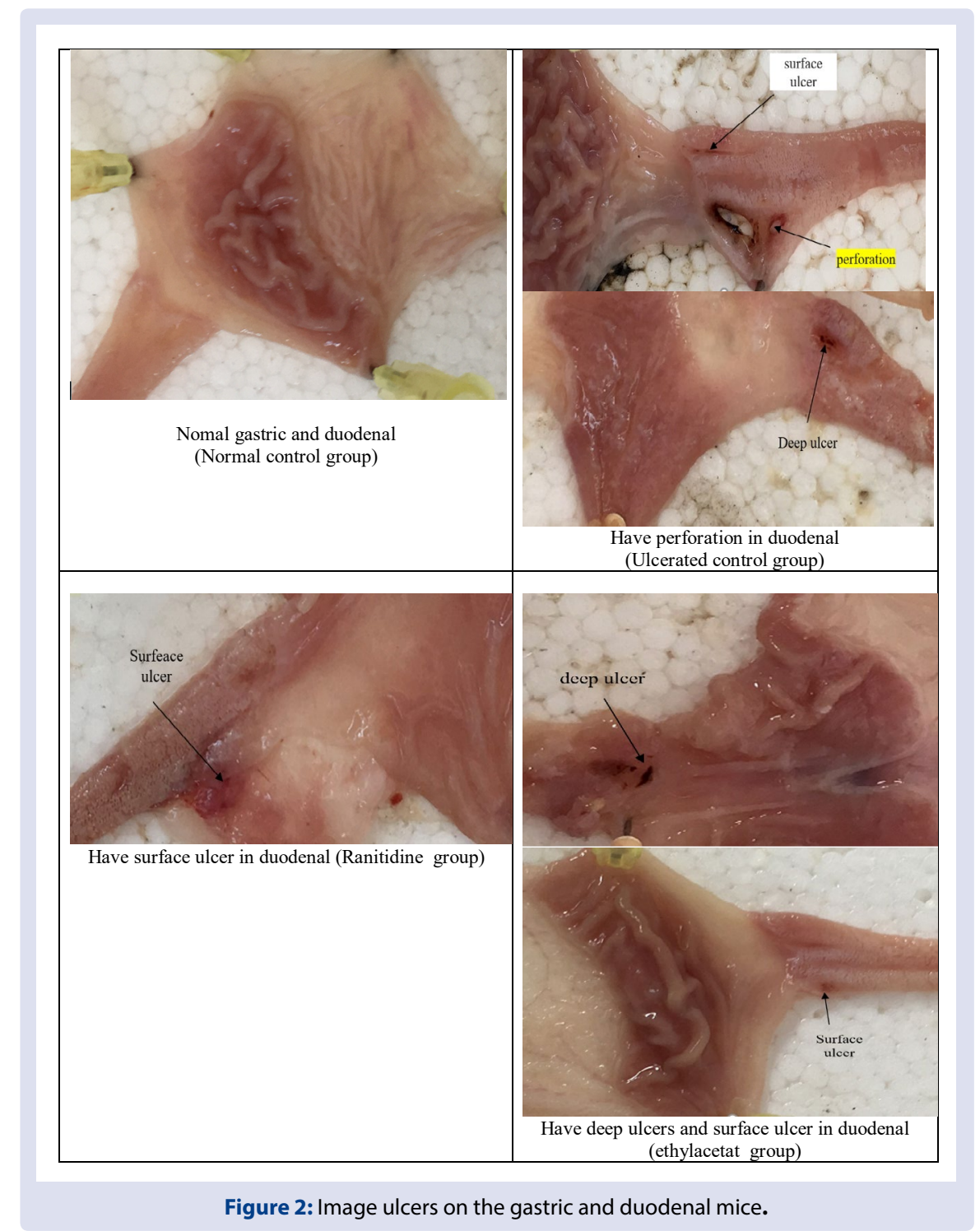

The adult Wistar albino rats were randomized into four groups of eleven rats with both the same sex ratio each

Group 1 (Normal control): distilled water $(10 \mathrm{ml} / \mathrm{kg})$

Group 2 (Ulcerated control): distilled water $(10 \mathrm{ml} / \mathrm{kg})+$ cysteamine $400 \mathrm{mg} / \mathrm{kg}$

Group 3 (Rantidine group): ranitidine $50 \mathrm{mg} / \mathrm{kg}+$ cysteamine $400 \mathrm{mg} /$ $\mathrm{kg}$

Group 4 (Ethylacetate group): ethylacetate extract $(11.52 \mathrm{mg} / \mathrm{kg}$ (according to folk experience equivalent dose for people, calculating with 6 conversion coefficients)) + cysteamine $400 \mathrm{mg} / \mathrm{kg}$

Treatments with the reference drug and extracts lasted for 10 days, on the 10th day, after one hour they were orally administered, groups 2, 3, 4 rats were orally administered twice daily with cysteamine $400 \mathrm{mg} / \mathrm{kg}$, at an interval of $4 \mathrm{~h}$. Rats were kept fasting before administering with cysteamine for 18 hours. $24 \mathrm{~h}$ after administration of the last dose of cysteamine, rats were anesthetized with thiopental to evaluate results.

Rats were showed stomach, opened duodenum and gastrointestinal tract by pulling along a large curve. Rinse with physiological saline, absorb surface ulcer with formaldehyde $5 \%$, fix duodenal stomach on foam sheet with pins. Ulcers were examined under 10-fold binocular magnification to assess lesions. Severity of gastric ulcer was assessed according to the point scale. ${ }^{12}$

\section{Evaluation index}

The average ulcer surface area $\left(\mathrm{mm}^{2}\right)$ : The transparent polyethylene film was placed on the ulcer surface. The outline traced onto the film using a permanent marker. An alternative to tracking was photographed, it was scanned image to create an image file by the scanner and the ulcer surface area area was calculated and measured the ImageJ Basic software (Version 1.38) by the National Institutes of Health and was recognized by the World Health Organization.

The ulcer index (UI):

$\mathrm{UI}=\mathrm{U}_{\mathrm{N}}+\mathrm{U}_{\mathrm{S}}+\mathrm{U}_{\mathrm{A}} \times 0,1$

Where UI is the ulcer index, UN is the ulcer number, US is the ulcer score and UA is the ulcer surface area.

\section{Statistical analysis}

All results are expressed as mean \pm SEM. Serial measurements were analyzed by using Two-way ANOVA with Tukey's post hoc test using SigmaStat 3.5 program and figures were performed by using SigmaPlot 10.0 program (Systat Software Inc). The critical significance level $\alpha$ was 0.050 and, then, statistical significance was defined as $P<0.05$. 


\section{RESULTS AND DISCUSSION}

Five identified compounds from the leave of Sanchezia nobilis Hook.F

\section{Compound 1: Quercetin}

Yellow powder, melting point range at $313 \div 314^{\circ} \mathrm{C}$. ESI-MS $\mathrm{m} / z 449$ $[\mathrm{M}+\mathrm{H}]^{+}$, Molecular formula $\mathrm{C}_{21} \mathrm{H}_{20} \mathrm{O}_{11}{ }^{1} \mathrm{H}-\mathrm{NMR},{ }^{13} \mathrm{C}-\mathrm{NMR}$ and DEPT data of compound 1 and reference data were in Table 1.

Compound 1 was isolated as yellow crystals, melting point range at 313 $\div 314^{\circ} \mathrm{C}$.

Two characteristic peaks was observed by ${ }^{1} \mathrm{H}-\mathrm{NMR}$ spectrum at $\delta_{\mathrm{H}} 6.19(1 \mathrm{H}, \mathrm{d}, J=2.0 \mathrm{~Hz}, \mathrm{H}-6)$ and $6.40(1 \mathrm{H}, \mathrm{d}, J=2.0 \mathrm{~Hz}, \mathrm{H}-8)$. Besides, three characteristic peaks was observed by ${ }^{1} \mathrm{H}-\mathrm{NMR}$ spectrum at 7.68 $\left(1 \mathrm{H}, \mathrm{d}, J=2.5 \mathrm{~Hz}, \mathrm{H}-2^{\prime}\right), 7.53\left(1 \mathrm{H}, \mathrm{dd}, J=8.5 ; 2.5 \mathrm{~Hz}, \mathrm{H}-6^{\prime}\right)$ and $6.88(1 \mathrm{H}$, d, $\left.J=8.5 \mathrm{~Hz}, \mathrm{H}-3^{\prime}\right)$ showed an ABX system.

The ${ }^{13} \mathrm{C}-\mathrm{NMR}$ and DEPT spectrum of compound $\mathbf{1}$ show the signal of $15 \mathrm{C}$ of the flavonoid frame in the middle of the area $\delta_{\mathrm{C}} 93.5 \div 175.9$ ppm, with $5 \mathrm{CH}$ aromatic ring in $\delta \mathrm{C} 98.3$ (C-6), 93.5 (C-8), 115.1 (C$\left.2^{\prime}\right), 115.8$ (C-5') and 120.1 (C-6'), in which 10 C signals $\delta \mathrm{C} 176.9 \mathrm{ppm}$ characterizes the carbonyl group, four carbon with displacement 145.2; $147.8 ; 160.9 ; 163.9 \mathrm{ppm}$ characterizes the bonding form of aromatic ring with $\mathrm{OH}$ group of carbon C-3', C-4, C-5, C-7. In addition, the signal of carbon at $\delta_{\mathrm{C}} 135.9(\mathrm{C}-3)$ characterizes the carbon of the double bond associated with a hydroxyl group.

Thus, the structure of $\mathbf{1}$ was assigned as quercetin that was consistent with the reported literature values. ${ }^{13}$

\section{Compound 2: Scopoletin}

Yellowish crystalline solid, melting point range at $203 \div 204^{\circ} \mathrm{C}$. ESI-MS $\mathrm{m} / z 193.1[\mathrm{M}+\mathrm{H}]^{+}$, Molecular formula $\mathrm{C}_{10} \mathrm{H}_{8} \mathrm{O}_{4}{ }^{1} \mathrm{H}-\mathrm{NMR},{ }^{13} \mathrm{C}-\mathrm{NMR}$ and DEPT data of compound 2 and reference data were in Table 2.

Compound 2 was isolated as yellowish crystalline solid, melting point range at $203 \div 204^{\circ} \mathrm{C}$. The mass spectra of ESI-MS for molecular mock ion with value $\mathrm{m} / \mathrm{z} 193.1[\mathrm{M}+\mathrm{H}]^{+}$confirm the molecular formula of compound 2 is $\mathrm{C}_{10} \mathrm{H}_{8} \mathrm{O}_{4}$.

${ }^{1} \mathrm{H}$-NMR spectrum shows signals of two separate doublets at $\delta_{\mathrm{H}} 6.26$ $(1 \mathrm{H}, \mathrm{d}, J=9.5 \mathrm{~Hz}, \mathrm{H}-3)$ and $7.60(1 \mathrm{H}, \mathrm{d}, J=9.5 \mathrm{~Hz}, \mathrm{H}-4)$ corresponding to 2 proton pyrone rings of coumarine. In the low field there are signals of 2 aromatic protons at $6.90(1 \mathrm{H}, \mathrm{s}, \mathrm{H}-5)$ and $6.90(1 \mathrm{H}, \mathrm{s}, \mathrm{H}-8) \mathrm{ppm}$. In addition, 1 signal of methoxy group appears at $\delta_{\mathrm{H}} 3.94\left(3 \mathrm{H}, \mathrm{s}, 6-\mathrm{CH}_{3}\right)$. ${ }^{13} \mathrm{C}$-NMR spectrum showed that compound 2 has $10 \mathrm{C}$, including signals at $\delta_{\mathrm{C}} 161.6(\mathrm{C}-2)$ and $150.4(\mathrm{C}-7)$ corresponding to carbon groups and phenolic carbonyl. The methoxy group also appeared at $\delta_{\mathrm{C}} 56.1 \mathrm{ppm}$. Spectral data also show that compound 2 has coumarin frame type. Combining spectral data of compound $\mathbf{2}$ with the reported literature values confirms compound $\mathbf{2}$ is scopoletin. ${ }^{14}$

\section{Table 1: NMR data of 1 and reference data.}

\begin{tabular}{|c|c|c|c|c|c|}
\hline Position C & DEPT & $\begin{array}{c}\delta_{\mathrm{C}}{ }^{1} \\
\mathrm{ppm}\end{array}$ & $\begin{array}{l}\delta_{C}{ }_{C}^{H a, b} \\
p p m\end{array}$ & $\begin{array}{c}\delta_{\mathrm{H}}{ }^{1}(\mathrm{ppm}) \\
\text { (Mult, J=Hz) }\end{array}$ & $\begin{array}{l}\delta_{\mathrm{H}}{ }^{\mathrm{Ha}, \mathrm{c}}(\mathrm{ppm}) \\
\text { (Mult, J=Hz) }\end{array}$ \\
\hline 2 & $\mathrm{C}$ & 146.8 & 147.2 & & \\
\hline 3 & $\mathrm{C}$ & 135.9 & 136.1 & & \\
\hline 4 & $\mathrm{C}$ & 175.9 & 176.2 & & \\
\hline 5 & $\mathrm{C}$ & 160.9 & 161.1 & & \\
\hline 6 & $\mathrm{CH}$ & 98.3 & 98.6 & $6.19(\mathrm{~d} ; 2.0)$ & $6.19(\mathrm{~d} ; 7.2)$ \\
\hline 7 & C & 163.9 & 164.3 & & \\
\hline 8 & $\mathrm{CH}$ & 93.5 & 93.8 & $6.40(\mathrm{~d} ; 2.0)$ & $6.41(\mathrm{~d} ; 6.9)$ \\
\hline 9 & C & 156.3 & 156.6 & & \\
\hline 10 & $\mathrm{C}$ & 103.2 & 103.4 & & \\
\hline $1^{\prime}$ & C & 122.2 & 122.4 & & \\
\hline $2^{\prime}$ & $\mathrm{CH}$ & 115.1 & 115.4 & $7.68(\mathrm{~d} ; 2.5)$ & $7.66(\mathrm{~d}, 7.4)$ \\
\hline $3^{\prime}$ & C & 145.2 & 145.5 & & \\
\hline $4^{\prime}$ & C & 147.8 & 148.1 & & \\
\hline 5 , & $\mathrm{CH}$ & 115.8 & 116.0 & $6.88(\mathrm{~d} ; 8.5)$ & $6.88(\mathrm{~d} ; 5.1)$ \\
\hline $6^{\prime}$ & $\mathrm{CH}$ & 120.1 & 120.4 & $7.53(\mathrm{dd} ; 8.5)$ & $7.54(\mathrm{~d} ; 6.9)$ \\
\hline
\end{tabular}

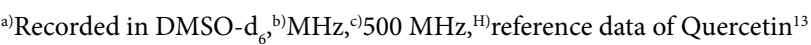

Table 2: NMR data of 2 and reference data.

\begin{tabular}{|c|c|c|c|c|c|}
\hline Position C & DEPT & $\begin{array}{l}\delta C 2 \\
\text { ppm }\end{array}$ & $\begin{array}{c}\delta C \text { la,b } \\
\text { ppm [6] }\end{array}$ & $\begin{array}{c}\delta \mathrm{H} 2 \text { (ppm) } \\
\text { (Mult, J=Hz) }\end{array}$ & $\begin{array}{c}\delta \mathrm{H} \text { la,c (ppm) } \\
\text { (Mult, J=Hz) [6] }\end{array}$ \\
\hline 2 & $\mathrm{C}$ & 161.6 & 162.6 & & \\
\hline 3 & $\mathrm{CH}$ & 107.6 & 108.1 & $6.26(\mathrm{~d} ; 9.5)$ & $6.20(\mathrm{~d} ; 9.2)$ \\
\hline 4 & $\mathrm{CH}$ & 143.4 & 144.2 & $7.60(\mathrm{~d} ; 9.5)$ & $7.70(\mathrm{~d} ; 9.0)$ \\
\hline 5 & $\mathrm{CH}$ & 113.4 & 111.6 & $6.90(\mathrm{~s})$ & $6.90(\mathrm{~s})$ \\
\hline 6 & $\mathrm{C}$ & 144.2 & 145.3 & & \\
\hline 7 & $\mathrm{C}$ & 150.4 & 151.0 & & \\
\hline 8 & $\mathrm{CH}$ & 103.1 & 102.9 & $6.90(\mathrm{~s})$ & $6.90(\mathrm{~s})$ \\
\hline 9 & $\mathrm{C}$ & 149.7 & 149.8 & & \\
\hline 10 & $\mathrm{C}$ & 111.4 & 110.9 & & \\
\hline 11 & $\mathrm{C}-\mathrm{O}-\mathrm{CH}_{3}$ & & & & $3.88(\mathrm{~s})$ \\
\hline
\end{tabular}

${ }^{\text {a) }}$ Recorded in $\mathrm{CDCl}_{3},{ }^{\text {b })} 125 \mathrm{MHz},{ }^{\text {c) }} 500 \mathrm{MHz}$, ${ }^{\text {I) }}$ reference of Scopoletin ${ }^{14}$ 
Compound 3: Kaempferol-3-O-a-L-rhamnopyranosyl- $(1 \rightarrow 6)-\beta-$ D-glucopyranoside

Yellow powder, $\alpha]^{20}=+11(\mathrm{c} 0.8, \mathrm{MeOH}) ; \mathrm{m}_{\mathrm{p}}=188-190^{\circ} \mathrm{C}, \mathrm{UV}$ $(\mathrm{MeOH}) \lambda_{\max }(\mathrm{nm}): 267,351 ; \mathrm{IR}(\mathrm{KBr}) v_{\max }\left(\mathrm{cm}^{-1}\right): 3410,1659,1605$, 1497, 1566, 1180, 1065; ESI-MS: $m / z 617.2[\mathrm{M}+\mathrm{Na}]^{+}, 593.2$ [M-H $^{-}$; Molecular formula: $\mathrm{C}_{27} \mathrm{H}_{30} \mathrm{O}_{15} ; \mathrm{M}=594 ;{ }^{1} \mathrm{H}-\mathrm{NMR},{ }^{13} \mathrm{C}-\mathrm{NMR}$ and DEPT data of compound 3 and reference data were in Table 3.

Compound 3 was isolated as Yellow powder, melting point range at $188-190^{\circ} \mathrm{C}$. UV spectroscopy $(\mathrm{MeOH})$ shows the specific absorption peaks for kaempferol glycoside derivatives at $\lambda_{\text {max }} 267$ and $351 \mathrm{~nm}$. IR spectra $(\mathrm{KBr})$ suggest the presence of hydroxyl groups $\left(3410 \mathrm{~cm}^{-1}\right)$, carbonyl $\left(1659 \mathrm{~cm}^{-1}\right)$, aromatic nuclei $\left(1605,1566,1497 \mathrm{~cm}^{-1}\right)$ and ether $\left(1180,1065 \mathrm{~cm}^{-1}\right)$. ESI-MS spectra of this compound for basic bases at $\mathrm{m} / \mathrm{z}$ 617.2; $[\mathrm{M}+\mathrm{Na}]^{+}, 593.2[\mathrm{M}-\mathrm{H}]$. Combined with ${ }^{13} \mathrm{C}-\mathrm{NMR}$ and DEPT spectral data, the molecular formula of the proposed compound 3 is $\mathrm{C}_{27} \mathrm{H}_{30} \mathrm{O}_{15}(\mathrm{M}=594 \mathrm{~g} / \mathrm{mol})$. The ${ }^{1} \mathrm{H}-\mathrm{NMR}$ spectrum of compound 3 indicates the characteristic signals of the flavone frame: 2 meta-protons of the A-ring at $\delta_{\mathrm{H}} 6.40(\mathrm{~s}, \mathrm{H}-8), 6.20(\mathrm{~s}, \mathrm{H}-6) ; 4$ aromatic protons of 1.4 B ring at $\delta_{\mathrm{H}} 8.10\left(\mathrm{~d}, J=9.0 \mathrm{~Hz}, \mathrm{H} 2-2^{\prime} / 6^{\prime}\right)$ and $6.89(\mathrm{~d}, J=9.0 \mathrm{~Hz}, \mathrm{H} 2-$ $\left.3^{\prime} / 5^{\prime}\right)$. The signal of the two protons anomer at $\delta_{\mathrm{H}} 5.08(\mathrm{~d}, J=8.0 \mathrm{~Hz}$, $\mathrm{H}-1$ "), 4.51 (s, H-1"') suggests the presence of two configurable sugar units $\beta$, $\alpha$ respectively. In addition, the signal of a second methyl group at $\delta_{\mathrm{H}} 1.18(\mathrm{~d}, J=6.5 \mathrm{~Hz}, \mathrm{H} 3-6$ "') was also recorded.

Analysis of ${ }^{13} \mathrm{C}-\mathrm{NMR}$ and DEPT spectra showed the presence of 27 carbons signals, including 1 methyl, 1 methylene carbon, 16 methine carbons and , 9 quaternary carbons.
In it, 15 carbon is assigned to the flavone frame and the remaining 12 carbon is assigned to 2 hexose units. Carbon signals at $\delta_{\mathrm{C}} 105.5$ (C1"), 73.8 (C-2"), 75.5 (C-3"), 70.2 (C-4"), 75.2 (C-5") and 67.5 (C6 ) indicates the presence of a $\beta$-Dglucopyranosyl unit. Meanwhile, the signal at $\delta_{\mathrm{C}} 101.9$ (C-1"'), 72.0 (C-2"'), 72.2 (C-3"'), 72.8 (C-4"'), 69.6 (C-5"') and 17.9 (C-6"') suggests the remaining sugar unit is a-Lrhamnopyranosyl. ${ }^{15}$ Road part signals are confirmed by $2 \mathrm{D}-\mathrm{NMR}$ spectrum analysis. In addition, HMBC interaction between $\mathrm{H}-1$ '” $\left(\delta_{\mathrm{H}}\right.$ 4.51) and C- 6 "' $\left(\delta_{C} 73.8\right)$ together with low field displacement of C-6 with corresponds to the normal value of $\beta$-D-glucopyranose. Proved that the binding order of sugar vessels is $\alpha$-Lrhamnopyranosyl- $(1 \rightarrow$ 6)- $\beta$-D-glucopyranoside. HMBC interactions between $\mathrm{H}-6 / \mathrm{H}-8$ and C-7 $\left(\delta_{\mathrm{C}} 166.1\right) / \mathrm{C}-10\left(\delta_{\mathrm{C}} 105.6\right)$, between H-6 and C-5 $\left(\delta_{\mathrm{C}} 162.8\right)$, between $\mathrm{H}-8$ and $\mathrm{C}-9(\delta \mathrm{C} 161.5)$, between $\mathrm{H}-2^{\prime} / 6^{\prime}$ and $\mathrm{C}-4$ ' $\left(\delta_{\mathrm{C}}\right.$ $159.5) / \mathrm{C}-2\left(\delta_{\mathrm{C}} 158.2\right)$, between $\mathrm{H}-3^{\prime} / 5^{\prime}$ and $\mathrm{C}-1^{\prime}\left(\delta_{\mathrm{C}} 122.5\right)$, together COSY H-2'/H-3' and H-5'/H-6 interactions allow the structure of the aglycone part of kaempferol to be established. In particular, HMBC interaction between $\mathrm{H}-1$ " $\left(\delta_{\mathrm{H}} 5.08\right)$ and C-3 $\left(\delta_{\mathrm{C}}\right.$ 135.6) confirms the binding position of the road section at $\mathrm{C}-3$ of the flavone frame. Based on the aforementioned data and combined with reference, compound 3 identified as kaempferol-3-O- $\alpha$-L-rhamnopyranosyl-( $1 \rightarrow 6)-\beta-D-$ glucopyranoside. ${ }^{15}$

Compound 4: Quercetin-3-O- $\alpha$-L-rhamnosyl- $(1 \rightarrow 6)-\beta-D$ glucopyranosyl-(1 $\rightarrow 3)-\beta-D$-glucopyranoside

Yellow solid, $m_{p}=191-193^{\circ} \mathrm{C} . \mathrm{R}_{\mathrm{f}}=0.24$ (TLC, silica gel, $\mathrm{n}$-butanol:acetic acid:water, 3:1:4, v/v/v), ESI-MS: m/z: 795.1 $[\mathrm{M}+\mathrm{Na}]^{+}, \mathrm{M}=772$,

Table 3: DEPT, ${ }^{1} \mathrm{H}$ - and ${ }^{13} \mathrm{C}-\mathrm{NMR}$ data of 3 and reference data.

\begin{tabular}{|c|c|c|c|c|c|}
\hline Position C & DEPT & $\begin{array}{c}\delta_{c}{ }^{3} \\
\mathrm{ppm}\end{array}$ & $\begin{array}{l}\delta_{c}^{\mathrm{La}, \mathrm{c}} \\
\mathrm{ppm} \\
\end{array}$ & $\begin{array}{c}\delta_{\mathrm{H}^{3}}(\mathrm{ppm}) \\
\text { (Mult, } \mathrm{J}=\mathrm{Hz} \text { ) }\end{array}$ & $\begin{array}{c}\delta_{H_{H}^{\mathrm{La}, \mathrm{c}}(\mathrm{ppm})} \\
\text { (Mult, J=Hz) }\end{array}$ \\
\hline 2 & $\mathrm{C}$ & 158.4 & 158.2 & - & - \\
\hline 3 & $\mathrm{C}$ & 135.6 & 135.4 & - & - \\
\hline 4 & C & 179.5 & 179.0 & - & - \\
\hline 5 & $\mathrm{C}$ & 162.8 & 162.7 & - & - \\
\hline 6 & $\mathrm{CH}$ & 100.0 & 99.9 & $6.20(\mathrm{~d} ; 1.5)$ & $6.19(\mathrm{~s})$ \\
\hline 7 & $\mathrm{C}$ & 166.1 & 165.7 & - & - \\
\hline 8 & $\mathrm{CH}$ & 94.8 & 94.8 & $6.40(\mathrm{~d} ; 1.5)$ & $6.37(\mathrm{~s})$ \\
\hline 9 & $\mathrm{C}$ & 161.5 & 159.2 & - & - \\
\hline 10 & $\mathrm{C}$ & 105.6 & 105.5 & - & - \\
\hline $1^{\prime}$ & $\mathrm{C}$ & 122.5 & 122.5 & - & - \\
\hline 2 & $\mathrm{CH}$ & 132.4 & 132.2 & $8.10(\mathrm{~d} ; 9.0)$ & $8.05(\mathrm{~d} ; 8.8)$ \\
\hline 3 & $\mathrm{CH}$ & 116.0 & 116.0 & $6.89(\mathrm{~d} ; 9.0)$ & $6.88(\mathrm{~d} ; 8.8)$ \\
\hline 4 & $\mathrm{CH}$ & 159.5 & 161.5 & $3.81(\mathrm{~s})$ & - \\
\hline 5 & $\mathrm{CH}$ & 116.0 & 116.0 & $6.88(\mathrm{~d} ; 9.0)$ & $6.88(\mathrm{~d} ; 8.8)$ \\
\hline 6 & $\mathrm{CH}_{2}$ & 132.4 & 132.2 & $8.10(\mathrm{~d} ; 9.0)$ & $8.05(\mathrm{~d} ; 8.8)$ \\
\hline $1^{\prime \prime}$ & $\mathrm{CH}^{2}$ & 105.5 & 104.6 & $5.08(\mathrm{~d} ; 8.0)$ & $5.11(\mathrm{~d} ; 7.2)$ \\
\hline $2^{\prime \prime}$ & $\mathrm{CH}$ & 73.8 & 75.6 & $3.32(\mathrm{~s})$ & $3.27-3.80$ \\
\hline $3^{\prime \prime}$ & $\mathrm{CH}$ & 75.5 & 78.2 & $3.62(\mathrm{~m})$ & $3.27-3.80$ \\
\hline $4^{\prime \prime}$ & $\mathrm{CH}$ & 70.2 & 73.8 & $3.80(\mathrm{~s})$ & $3.27-3.80$ \\
\hline $5^{\prime \prime}$ & $\mathrm{CH}$ & 75.2 & 78.0 & $3.56(\mathrm{~m})$ & $3.27-3.80$ \\
\hline $6^{\prime \prime}$ & $\begin{array}{l}\mathrm{CH} \\
\mathrm{CH}\end{array}$ & 67.5 & 68.5 & $\begin{array}{l}3.40(\mathrm{~m}) \\
3.71(\mathrm{~m})\end{array}$ & $3.27-3.80$ \\
\hline $1^{\prime \prime \prime}$ & $\mathrm{CH}$ & 101.9 & 102.2 & $4.51(\mathrm{~s})$ & $4.51(\mathrm{~s})$ \\
\hline $2^{\prime \prime \prime}$ & $\mathrm{CH}$ & 72.0 & 71.9 & $3.64 \mathrm{~m})$ & $3.25-3.81$ \\
\hline $3^{\prime \prime \prime}$ & $\mathrm{CH}$ & 72.2 & 72.2 & $3.56(\mathrm{~m})$ & $3.25-3.81$ \\
\hline $4^{\prime \prime \prime}$ & $\mathrm{CH}$ & 72.8 & 71.3 & $3.81(\mathrm{~m})$ & $3.25-3.81$ \\
\hline $5^{\prime \prime \prime}$ & $\mathrm{CH}$ & 69.6 & 69.6 & $3.55(\mathrm{~m})$ & $3.25-3.81$ \\
\hline 6 & $\mathrm{CH}_{3}$ & 17.9 & 18.0 & $1.18(\mathrm{~d} ; 6.0)$ & $1.12(\mathrm{~d} ; 6.0)$ \\
\hline
\end{tabular}

${ }^{a}$ ) Recorded in $\mathrm{CD}_{3} \mathrm{OD},{ }^{\mathrm{b})} 100 \mathrm{MHz},{ }^{\mathrm{c})} 400 \mathrm{MHz}$, ${ }^{\mathrm{L})}$ reference data of Kaempferol-3-O- $\alpha$-L-rhamnopyranosyl-( $(1 \rightarrow 6)-\beta-\mathrm{D}$-glucopyranoside ${ }^{15}$ 
Table 5: DEPT, ${ }^{1} \mathrm{H}-\mathrm{và}{ }^{13} \mathrm{C}-\mathrm{NMR}$ data of compound 5 and the reference.

\begin{tabular}{|c|c|c|c|c|c|}
\hline Position C & DEPT & $\begin{array}{c}\delta_{\mathrm{C}}{ }^{4} \\
\mathrm{ppm}\end{array}$ & $\begin{array}{l}\delta_{\mathrm{c}}^{\mathrm{Ma}, \mathrm{b}} \\
\mathrm{ppm}\end{array}$ & $\begin{array}{c}\delta_{H}{ }^{4}(\mathrm{ppm}) \\
\text { (Mult, J=Hz) }\end{array}$ & $\begin{array}{l}\delta_{\mathrm{H}}^{\mathrm{Ma}, \mathrm{c}}(\mathrm{ppm}) \\
\text { (Mult, } \mathrm{J}=\mathrm{Hz} \text { ) }\end{array}$ \\
\hline 2 & $\mathrm{C}$ & 158.4 & 156.5 & - & - \\
\hline 3 & $\mathrm{C}$ & 134.8 & 133.3 & - & - \\
\hline 4 & $\mathrm{C}$ & 179.5 & 177.3 & - & - \\
\hline 5 & $\mathrm{C}$ & 163.0 & 161.2 & - & - \\
\hline 6 & $\mathrm{CH}$ & 99.8 & 98.7 & $6.20(\mathrm{~d} ; 2.0)$ & $6.19(\mathrm{~d} ; 2.0)$ \\
\hline 7 & $\mathrm{C}$ & 165.8 & 164.2 & - & - \\
\hline 8 & $\mathrm{CH}$ & 94.8 & 93.6 & $6.40(\mathrm{~d} ; 2.0)$ & $6.38(\mathrm{~d} ; 2.0)$ \\
\hline 9 & $\mathrm{C}$ & 159.3 & 156.4 & - & - \\
\hline 10 & $\mathrm{C}$ & 105.8 & 103.9 & - & - \\
\hline $1^{\prime}$ & $\mathrm{C}$ & 123.1 & 121.1 & - & - \\
\hline 2 & $\mathrm{CH}$ & 116.1 & 115.2 & $7.61(\mathrm{~d} ; 2.0)$ & $7.53(\mathrm{~d} ; 8.2)$ \\
\hline 3 & C & 145.9 & 144.7 & - & - \\
\hline 4 & C & 149.7 & 148.4 & - & - \\
\hline 5 & $\mathrm{CH}$ & 117.6 & 116.2 & $6.90(\mathrm{~d} ; 8.5)$ & $6.84(\mathrm{~d} ; 8.2)$ \\
\hline 6 & $\mathrm{CH}$ & 123.1 & 121.6 & $7.54(\mathrm{dd} ; 8.5 ; 2.0)$ & $7.53(\mathrm{~d} ; 8.2)$ \\
\hline $1^{\prime \prime}$ & $\mathrm{CH}$ & 101.1 & 101.2 & $5.31(\mathrm{~d} ; 7.5)$ & $5.34(\mathrm{~d} ; 7.3)$ \\
\hline $2^{\prime \prime}$ & $\mathrm{CH}$ & 77.0 & 74.1 & $3.32(\mathrm{~m})$ & \\
\hline $3^{\prime \prime}$ & $\mathrm{CH}$ & 82.5 & 76.4 & $3.76(\mathrm{~s})$ & \\
\hline $4^{\prime \prime}$ & $\mathrm{CH}$ & 71.0 & 67.0 & $3.45(\mathrm{~m})$ & \\
\hline $5 "$ & $\mathrm{CH}$ & 77.8 & 75.9 & $3.41(\mathrm{~m})$ & \\
\hline $6 a$ & $\mathrm{CH}$ & 62.5 & 67.0 & $3.73(\mathrm{dd} ; 11.5 ; 5.0)$ & \\
\hline 6"b & $\mathrm{CH}$ & 62.5 & 67.0 & $3.82(\mathrm{dd} ; 1.5 ; 2.0)$ & \\
\hline $1 "$ & $\mathrm{CH}$ & 104.8 & & $4.73(\mathrm{~d} ; 7.5)$ & $4.38(\mathrm{~s})$ \\
\hline $2^{\prime \prime \prime}$ & $\mathrm{CH}$ & 75.6 & & $3.41(\mathrm{~m})$ & \\
\hline $3^{\prime \prime \prime}$ & $\mathrm{CH}$ & 78.2 & & $3.35(\mathrm{~m})$ & \\
\hline $4^{\prime \prime \prime}$ & $\mathrm{CH}$ & 71.4 & & $3.32(\mathrm{~m})$ & \\
\hline $5^{\prime \prime \prime}$ & $\mathrm{CH}$ & 77.9 & & $3.60(\mathrm{~m})$ & \\
\hline $6 \mathrm{a}$ & $\mathrm{CH}$ & 68.2 & & $3.36(\mathrm{~s})$ & \\
\hline 6"'b & $\mathrm{CH}$ & 68.2 & & $3.80(\mathrm{~m})$ & \\
\hline $1^{\prime \prime \prime}$ & $\mathrm{CH}$ & 102.1 & 101.7 & $4.52(\mathrm{~d} ; 1.0)$ & \\
\hline $2^{m+m}$ & $\mathrm{CH}$ & 72.2 & 70.3 & $3.50(\mathrm{dd} ; 9.5 ; 3.5)$ & \\
\hline $3^{\prime \prime \prime}$ & $\mathrm{CH}$ & 72.0 & 70.5 & $3.60(\mathrm{~m})$ & \\
\hline $4^{\prime \prime \prime}$ & $\mathrm{CH}$ & 73.8 & 71.8 & $3.27(\mathrm{t} ; 9.5)$ & \\
\hline $5^{\prime \prime \prime}$ & $\mathrm{CH}$ & 69.8 & 68.2 & $3.44(\mathrm{~m})$ & \\
\hline 6 & $\mathrm{CH}_{3}$ & 17.7 & 17.7 & $1.10(\mathrm{~d} ; 6.5)$ & $0.99(\mathrm{~d} ; 6.2)$ \\
\hline
\end{tabular}

${ }^{\text {a) }}$ recorded in $\mathrm{CDCl}_{3},{ }^{\mathrm{b})} 125 \mathrm{MHz},{ }^{\mathrm{c}} 500 \mathrm{MHz},{ }^{\mathrm{N})}$ the reference: 3'-O-methyl-3,4- methylenedioxyellagic acid ${ }^{17}$

${ }^{1} \mathrm{H}-\mathrm{NMR},{ }^{13} \mathrm{C}-\mathrm{NMR}$, DEPT data of compound $\mathbf{4}$ and reference compound are shown in Table 4.

Yellow solid, melting temperature is about $191-193^{\circ} \mathrm{C} . \mathrm{R}_{\mathrm{f}}=0.24$ (TLC, silica gel, n-butanol:acetic acid:water, 3:1:4, v/v), ESI-MS: m/z: 795.1 $[\mathrm{M}+\mathrm{Na}]^{+}, \mathrm{M}=772$, Molecular formula: $\mathrm{C}_{33} \mathrm{H}_{40} \mathrm{O}_{21}$

${ }^{1} \mathrm{H}-\mathrm{NMR}$ and ${ }^{13} \mathrm{C}-\mathrm{NMR}$ data of compound 4 have characteristics of a flavonoid glycoside. Aglycon part is defined as quercetin. When we compare the carbon number of compound $\mathbf{4}$ with quercetin, it is possible to have 3 sugar units in 152 structures of this compound. Based on the analysis of $1 \mathrm{D}-\mathrm{NMR}$ data, it is possible to have 2 $\beta$-glucose units and one $\alpha$-L-rhamnose units. The binding position between the sugar units and the quercetin and between the sugar units and each others is determined based on the HMBC spectrum. HMBC spectrum showed the interaction of $\beta$-glucose's anomeric proton at $\delta_{\mathrm{H}} 5.31\left(1 \mathrm{H}, \mathrm{d}, J=7.5 \mathrm{~Hz}, \mathrm{H}-1\right.$ ") with carbon $\delta_{\mathrm{C}} 134.8(\mathrm{C}-$ 3 ) so this $\beta$-glucose binds with quercetin by 3 -O-glycoside link. A noteworthy point is appearance of shift peak at $\delta_{\mathrm{C}} 82.5$ (C-3"), which interacts with the second glucose's anomeric proton at $\delta_{\mathrm{H}} 4.73(1 \mathrm{H}, \mathrm{d}$, $J=7.5 \mathrm{~Hz}, \mathrm{H}-1$ "') in the carbon resonance region of the sugar. It shows the link $(1 \rightarrow 3)$-glucosid between these two sugars. Further analysis of the HMBC correlation between the rhamnose and the quercetin and the two sugars show that this sugar must be linked to the second glucose by $(1 \rightarrow 6)$-glycoside link because of the interaction between the proton $\delta_{\mathrm{H}} 4.52\left(1 \mathrm{H}, \mathrm{d}, J=1.0 \mathrm{~Hz}, \mathrm{H}-1^{\prime \prime \prime \prime}\right)$ with carbon $\delta_{\mathrm{C}} 68.2$ $(\mathrm{C}-6$ "). Based on the above analysis in conjunction and the reference, the structure of compound 4 is quercetin-3-O- $\alpha$-L-rhamnosyl- $(1 \rightarrow 6)$ $\beta$-D-glucopyranosyl-( $(1 \rightarrow 3)-\beta$-D-glucopyranoside. ${ }^{16}$

\section{Compound 5: 3'-O-methyl-3,4-methylenedioxy ellagic acid}

White solid, $\mathrm{m}_{\mathrm{p}}=296-298{ }^{\circ} \mathrm{C} . \mathrm{R}_{\mathrm{f}}=0.51$ (TLC, silica gel, dichloromethane:methanol, 20:1, v/v). ${ }^{1} \mathrm{H}-\mathrm{NMR},{ }^{13} \mathrm{C}-\mathrm{NMR}$, DEPT data of compound 5 and the reference compound are shown in Table 5.

White solid, melting temperature about $296-298^{\circ} \mathrm{C} . \mathrm{R}_{\mathrm{f}}=0.51$ (TLC, silica gel, diclometan:methanol, 20:1, v/v), showing light pink color with $1 \%$ vanillin/sulfuric acid reagent.

${ }^{1} \mathrm{H}-\mathrm{NMR}$ spectrum of 5 shows only 2 singlet signals at $\delta_{\mathrm{H}} 7.52(1 \mathrm{H}$, s, $\left.\mathrm{H}_{-} 5^{\prime}\right)$ and $7.51(1 \mathrm{H}, \mathrm{s}, \mathrm{H}-5)$. They are defined as two protons of the benzene ring. Singlet signals at $\delta_{\mathrm{H}} 6.36(2 \mathrm{H}, \mathrm{s}, \mathrm{H}-3 \mathrm{a})$ showed the presence of two methylenedioxy protons. In addition, the methoxy group is determined by the signal at $\delta_{\mathrm{H}} 4.04(3 \mathrm{H}, \mathrm{s})$. 
${ }^{13} \mathrm{C}$-NMR and DEPT of compound $\mathbf{5}$ showed that this compound has 15 C, in which $12 \delta_{C}$ signals 110.9 (C-1'), $112.2\left(\mathrm{C}-5^{\prime}\right), 103.8(\mathrm{C}-5), 116.1$ (C-6'), 116.1 (C-6), 112.8 (C-1), 140.2 (C-3'), 131.1 (C-2), 138.2 (C-3), 141.5 (C-2'), 150.1 (C-4), 150.1 (C-4') belong to two benzene rings with $10 \mathrm{C}$ and $2 \mathrm{CH}$. In addition, two carbonyl carbon signals appeared at $\delta_{\mathrm{C}} 157.5$ (C-6’a), 158.2 (C-6a), which are very specific for esters ring (lactones) in derivativesof ellagic acid. The presence of the $\mathrm{CH}_{2}$ group linked with 2 atoms $\mathrm{O}$ and the methoxy group in turn at $\delta \mathrm{C} 104.2(\mathrm{C}$ 3a) and $60.8\left(\mathrm{~s}, 3-\mathrm{OCH}_{3}\right)$.

Based on the above analysis and compared with the previously published spectral data the structure of substance 5 was determined to be 3'-O-methyl-3,4-methylenedioxyellagic acid. ${ }^{17}$

\section{Anti-ulcer activity}

\section{Anti-duodenal ulcer effect of ethylacetate extract samples on the model of duodenal ulcer caused by cysteamine}

The results in Table 6 showed:

- Normal control group has no ulcer image in all mice.

- The rate of mice with ulcers in the Ulcerated control group is $100 \%$.

- Ranitidine group significantly reduced the incidence of cysteamine ulcers compared to the Ulcerated control group, the difference was statistically significant with $p(X>\chi 2)=0.044(\alpha=0.05)$

- Ethylacetate group has ulcer rate of $100 \%$, no difference from the mock-up group.
The results in Table 7 showed:

- Normal control group had no ulcer image in all mice.

- Ulcerated control group had damage levels including surface ulcers, deep ulcers and perforation, in which deep ulcers are the main lesion at the rate of $62.50 \%$.

- Ranitidine group had damage levels including surface ulcers and deep ulcers, in which surface ulcers are the main lesion at $57.14 \%$. The prevalence of deep ulcers in the batch of ranitidine ( $42.86 \%$ ) decreased by 1.46 times compared to the Ulcerated control group (62.50\%). There was no ulceration in rats given ranitidine.

- Ethylacetate group had damage levels including surface ulcers and deep ulcers, no ulcer perforation. Deep ulcer is a higher rate of injury in both study samples (54.17\%), but this rate has decreased compared to the Ulcerated control group (62.50\%).

The data in Table 8 showed:

- Ranitidine dose of $50 \mathrm{mg} / \mathrm{kg}$ significantly reduced the ulcer score compared to the mock-up group, the difference was statistically significant with $p=0.001$.

- The ulcer score in the mice group taking samples of ethylacetate fraction - There is no difference in ulcer surface area between ranitidine oral groups and ethylacetate extract reagents compared to mock-up groups $(p>0.05)$.

Table 5: DEPT, ' $\mathrm{H}$ - và ${ }^{13} \mathrm{C}-\mathrm{NMR}$ data of compound 5 and the reference.

\begin{tabular}{|c|c|c|c|c|c|}
\hline Vị trí C & DEPT & $\begin{array}{c}\delta_{c}^{5} \\
\mathrm{ppm}\end{array}$ & $\begin{array}{l}\delta_{C}^{\mathrm{Na}, \mathrm{b}} \\
\mathrm{ppm}\end{array}$ & $\begin{array}{c}\delta_{\mathrm{H}}{ }^{5}(\mathrm{ppm}) \\
\text { (Mult, } \mathrm{J}=\mathrm{Hz} \text { ) }\end{array}$ & $\begin{array}{l}\delta_{H} \mathrm{Na}, \mathrm{C}^{(p p m)} \\
\text { (Mult, J=Hz) }\end{array}$ \\
\hline 1 & $\mathrm{CH}_{2}$ & 112.8 & 116.0 & & \\
\hline 2 & $\mathrm{C}$ & 131.1 & 131.0 & - & - \\
\hline 3 & $\mathrm{C}$ & 138.2 & 138.2 & - & - \\
\hline $3 a$ & $\mathrm{CH}_{2}$ & 104.2 & 104.2 & $6.36(\mathrm{~s})$ & $6.37(\mathrm{~s})$ \\
\hline 4 & C & 150.1 & 149.9 & - & - \\
\hline 5 & $\mathrm{CH}$ & 103.8 & 103.8 & $7.51(\mathrm{~s})$ & $7.52(\mathrm{~s})$ \\
\hline 6 & $\mathrm{C}$ & 116.1 & 110.9 & - & - \\
\hline $6 a$ & C & 158.2 & 158.2 & - & - \\
\hline 1 & $\mathrm{C}$ & 110.9 & 111.0 & - & - \\
\hline 2 & $\mathrm{C}$ & 141.5 & 141.5 & - & - \\
\hline 3 & $\mathrm{C}$ & 140.2 & 140.2 & - & - \\
\hline 3'- $-\mathrm{OCH}_{3}$ & $\mathrm{CH}_{3}$ & 60.8 & 60.8 & $4.04(\mathrm{~s})$ & $4.04(\mathrm{~s})$ \\
\hline 4 & $\mathrm{C}$ & 150.1 & 152.9 & - & - \\
\hline 5 & $\mathrm{CH}$ & 112.2 & 112.1 & $7.52(\mathrm{~s})$ & $7.53(\mathrm{~s})$ \\
\hline 6 & C & $\begin{array}{l}116.1 \\
157.5\end{array}$ & $\begin{array}{c}1126 \\
157.6\end{array}$ & - & - \\
\hline
\end{tabular}

${ }^{a)}$ recorded in $\mathrm{CDCl}_{3}$, ${ }^{\mathrm{b})} 125 \mathrm{MHz},{ }^{\mathrm{c})} 500 \mathrm{MHz},{ }^{\mathrm{N})}$ the reference: 3'-O-methyl-3,4- methylenedioxyellagic acid ${ }^{17}$

Table 6: Percentage of mice with ulcer images in the study groups.

\begin{tabular}{ccc}
\hline & Percentage of mice with ulcer images (\%) & Percentage of mice without ulcer images (\%) \\
\hline Normal control group & 0 & 100 \\
Ulcerated control group & 100 & 0 \\
Ranitidine group & 72.7 & 27.3 \\
Ethylacetate group & 100 & 0
\end{tabular}

Table 7: Effect of reagent samples on the severity of gastric-duodenal lesions.

\begin{tabular}{cccc}
\hline & Perforation (\%) & deep ulcer (\%) & surface ulcer (\%) \\
\hline Normal control group & 0 & 0 & 0 \\
Ulcerated control group & 6.25 & 62.50 & 31.25 \\
Ranitidine group & 0 & 42.86 & 57.14 \\
Ethylacetate group & 0 & 54.17 & 45.83 \\
\hline
\end{tabular}


Table 8: Effects of reagent samples on ulcer score, ulcer surface area and ulcer index.

\begin{tabular}{|c|c|c|c|c|}
\hline Group & $\mathrm{n}$ & ulcer score & ulcer surface area $(\mathrm{mm} 2)$ & ulcer index \\
\hline Ulcerated control group & 13 & $2.46 \pm 0.52$ & $5.28 \pm 1.79$ & $8,07 \pm 2,21$ \\
\hline Ranitidine group & 11 & $1.27 \pm 0.90^{* * *}$ & $4.01 \pm 2.91$ & $3,77 \pm 2,63^{* * *}$ \\
\hline Ethylacetate group & 13 & $1.85 \pm 0.80^{*}$ & $5.39 \pm 1.94$ & $5,61 \pm 2,69^{*}$ \\
\hline
\end{tabular}

${ }^{*} p<0.05 ;{ }^{* *} p<0.001$ compair with mock-up group (Mann-Whitney test)

- Ranitidine dose of $50 \mathrm{mg} / \mathrm{kg}$ significantly reduced ulcer index compared to the mock-up group, the difference was statistically significant with $\mathrm{p}=0.001$.

- Significant index of ulcer in mouse group taking sample of ethylacetate extract compared with mock-up group with $\mathrm{p}=0.04$.

\section{DISCUSSION}

Five substances were extracted and structurally determined from the ethylacetate extract of Sanchezia nobilis Hook.F. leaves in which compound 1.2 was published. Four of them are flavonoids. Flavonoids has a good antioxidant and anti-inflammatory effect, especially quercetin the most important mechanism of action responsible for the anti-ulcer activity of flavonoids is their antioxidant properties, quercetin, which involves free radical scavenging, transition metal ions chelation, inhibition of oxidizing enzymes, increase of proteic and nonproteic antioxidants and reduction of lipid peroxidation. Quercetin (flavanones) accelerate the healing of gastric ulcers. ${ }^{18}$ Besides, Quercetin compound has been shown to have anti-cancer, anti-inflammatory and inhibition of bacteria, some strains of viruses and fungi ... ${ }^{19-21}$ This may explain the effect of anti-duodenal ulcer in the segment of the leaves of etheyacetate extract of Sanchezia nobilis Hook.F. In the past, there has been no announcement about this effect of plants.

\section{CONCLUSION}

From the ethylacetate extract of Sanchezia nobilis Hook.F. leaves collected in Nam Dinh province, Vietnam, extracted and determined the structure of 5 compounds: Quercetin (1), Scopoletin (2), Kaempferol-3-O- $\alpha$-L-rhamnopyranosyl- $(1 \rightarrow 6)-\beta$-D-glucopyranoside (3), Quercetin-3-O- $\alpha$-L-rhamnosyl- $(1 \rightarrow 6)-\beta$-D-glucopyranosyl- $(1 \rightarrow 3)$ - $\beta$-D-glucopyranosid (4), 3'-O-methyl-3,4-methylenedioxy ellagic acid (5). In which compounds $3,4,5$ are the first isolated from Sanchezia nobilis Hook.F.

The extract of ethylacetate from leaves of Sanchezia nobilis Hook.F. has been tested against the effect of duodenal ulcer on cysteamine duodenal ulcer model with dose equivalent to human dose, showing that the extract improves the number of ulcers, reduces the ulcer score and the ulcer index, however, there is no change in ulcer surface area compared to control group. But the effect is not as good as ranitidine group.

\section{ACKNOWLEDGEMENT}

The research was supported by has been financed by Vietnam National University, Hanoi with grants number: QG.18.20

\section{CONFLICTS OF INTERESTS}

These authors have declared that there is no conflicts of interest.

\section{REFERENCES}

1. Md. Abu SR, Parvin S, Md. Abdul K, Sharmin T. Preliminary phytochemical screening and cytotoxic potentials from leaves of Sanchezia speciosa Hook. International Journal of Advances in Scientific Research. 2015;1(3):145-50.
2. Parvin S, Rafshanjani AS, Md Abdul K. In vitro antibacterial, antifungal and insecticidal activities of ethanolic extract and its fractionates of Sanchezia speciosa Hook. Int Res J Pharm. 2004;5(9):717-20.

3. Paydar M, Wong YL, Moharam BA, Wong WF, Looi CY. In vitro anti-oxidant and anti-cancer activity of methanolic extract from Sanchezia speciosa leaves. Pakistan Journal of Biological Sciences. 2013;16(20):1212-5.

4. Shaheen N, Uzair M, Ch BA, Alamgeer. In vitro cytotoxicity of Sanchezia speciosa extracts on human epithelial cervical cancer (hela) cell line. Acta Poloniae Pharmaceutica - Drug Research. 2017;1389-94.

5. Tung VDLBT, Hai NT, Vung NT. In nitro antioxidant and anti-inflammatory activities of isolated compound of ethanol eaxtra from Sanchezia speciosa Leonard's leaves. Journal of Basic and Clinical Physiology and Pharmacology. 2017;28(1):79-84

6. Ellah AEA, Mohamed KM, Backheet EY, Mohamed MH. Matsutake alcohol glycosides from Sanchezia nobilis. Chemistry of Natural Compounds. 2013;48(6):930-3.

7. Omondi S, Omondi JC. Phytochemical analysis of 50 selected plants found in the University Botanic Garden, Maseno, Kenya for their chemotaxonomic values. Journal of Medicinal Herbs and Ethnomedicine. 2015;1:130-5.

8. Ellah AEA, Mohamed KM, Backheet EY, Mohamed MH. Cinnamyl alcohol, benzyl alcohol and flavonoid glycosides from Sanchezia nobilis. Chemistry of Natural Compounds.2014;823-6.

9. Hoang HP. Viet Nam Vegetation. Young publisher. 2000;39.

10. Elberry AA. Protective effect of sildenafil against cysteamine induced duodenal ulcer in Wistar rats. African Journal of Pharmacy and Pharmacology. 2013;7(33):2352-7.

11. Rezvanjoo B, Rashidi S, Jouyban A, Beheshtiha SH, Samini M. Effects of vitamin $\mathrm{C}$ and melatonin on cysteamine induced duodenal ulcer in a cholestatic rat model: A controlled experimental study. Current Therapeutic Research. 2010;71(5):322-30.

12. Vogel HG, Hock FJ, Maas J, Mayer D. Drug discovery and evaluation (Pharmacological Assays). Berlin: SpringerVerlag. 1997;486-7.

13. Sambandam B, Thiyagarajan D, Ayyaswamy A, Raman P. Extraction and isolation of flavonoid Quercetin from the leaves of Trigonella foenum-graecum and their anti-oxidant activity. International Journal of Pharmacy and Pharmaceutical Sciences. 2016;8(6):120-4.

14. Khan NMMU, Hossain Md. S. Scopoletin and $\beta$-sitosterol glucoside from roots of Ipomoea digitata. Journal of Pharmacognosy and Phytochemistry. 2015;5-7.

15. Han JT, Bang MH, Chun OK, Kim DO, Lee CY, Baek MI. Flavonol glycosides from the aerial parts of Aceriphyllum rossii and their antioxidant activities. Arch Pharm. 2004;390-5.

16. Moustafa AMY, Khodair Al, Saleh MA. Isolation, structural elucidation of flavonoid constituents from Leptadenia pyrotechnica and evaluation of their toxicity and antitumor activity. Pharmaceutical Biology. 2009;539-52.

17. Khallouki F, Haubner $R$, Hull WE, Erben G, Spiegelhalder B, Bartsch $H$, et al. Isolation, purification and identification of ellagic acid derivatives, catechins and procyanidins from the root bark of Anisophyllea dichostyla R. Br. Science Direct. 2007;472-85.

18. Mota KSdeL, Dias GEN, Pinto MEF, Ferreira ML, Brito ARMS, Lima CAH, et al. Flavonoids with gastroprotective activity. Molecules. 2009;14:979-1012.

19. Pereira MA, Grubbs CJ, Barnes LH, Li H, Olson GR, Eto I, et al. Effects of the phytochemicals, curcumin and quercetin, upon azoxymethane-induced colon cancer and 7,12-dimethylbenz[a]anthracene-induced mammary cancer in rats. Carcinogenesis. 1996;17(6):1305-11.

20. Joshi UJ, D'Mello GAS, Sudha RS. Anti-inflammatory, antioxidant and anticancer activity of quercetin and its analogues. International Journal of Research in Pharmaceutical and Biomedical Sciences. 2011;2(4):1756-66.

21. Shu Y, LY, Li L, Feng J, Lou B, Zhou X, Wu H. Antibacterial activity of quercetin on oral infectious pathogens. African Journal of Microbiology Research. 2011;5(30):5358-61. 


\section{GRAPHICAL ABSTRACT}

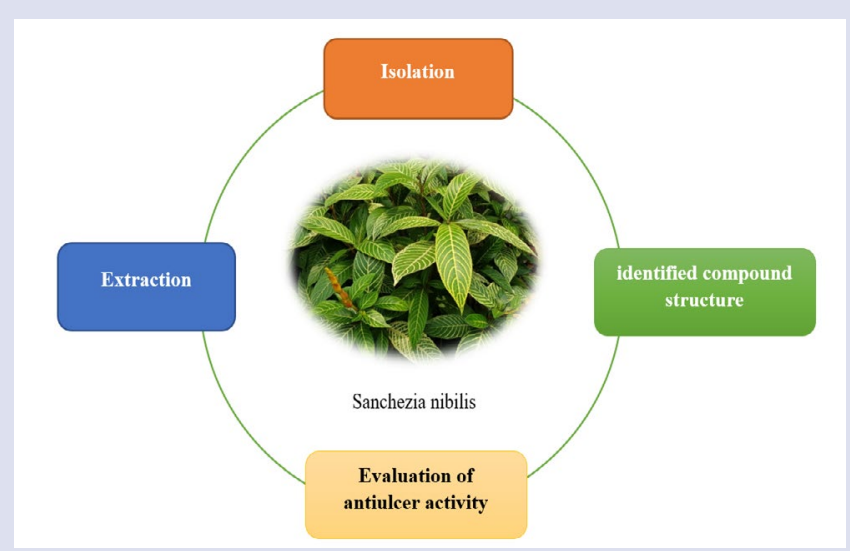

\section{SUMMARY}

All the demonstrated data revealed that five compounds were extracted, isolated from the leaves of Sanchezia nobilis. Furthermore, evaluation the effect of gastric and duodenal anti-ulcer on cyteamine induced gastric ulcer models indicates at this fractional ethylacetate has the effect to improve the extent of ulcer lesions treatment.

\section{ABOUT AUTHORS}

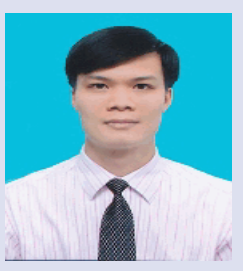

Dr. Vu Duc Loi is currently the Head of Department of Pharmacognosy and Traditional Pharmacy, School of Medicine and Pharmacy, Viet nam National University, Hanoi, Vietnam. He has over 12 years of experience in teaching Phytochemistry, Pharmacognosy and Medicinal Plant. He is specialized in chemistry of natural products with a special interest in terpenoid, flavonoids and alkaloids. He has published more than 90 scientific articles.

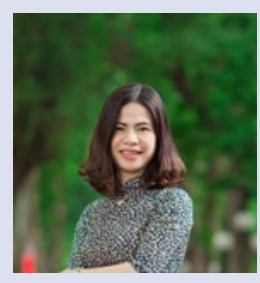

M.Sc BuiThi Xuan is a lecture from School of Medicine and Pharmacy, Vietnam National University. She has been teaching, researching for more than 10 years, published many scientific papers and Phytochemistryoriented scientific research topics. She is studying for a PhD in medicine plant. The study focused on finding new substances in plants and testing the biological effects of different extracts.

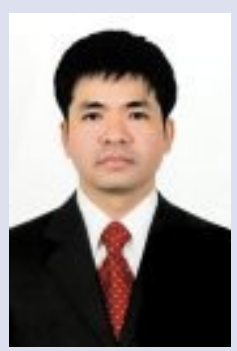

Tran Minh Ngoc Ph.D, Vice - Director of National Institute of Medicinal Material, Hanoi, Vietnam. He has experience in modern spectroscopy and structural elucidation of natural products using 1D and 2D NMR (HSQC, HMBC, COSY, ROESY and NOESY) technique, as well as MS (HRMS, GC-MS, LC-ESI-MS) analysis and experience in a protocol for traditional \& modern medicine, and marker compounds in pharmaceutical material medicine plant. He has published more than 30 scientific articles.

Cite this article: Duc LV, Thi XB, Minh NT. Chemical constituents and anti-ulcer activity of ethylacetate extract of the leaves of Sanchezia nobilis Hook.F. Pharmacog J. 2019;11(6):1172-80. 